\title{
Extended Conway-Maxwell-Poisson distribution and its properties and applications
}

\author{
Subrata Chakraborty ${ }^{1^{*}}$ and Tomoaki Imoto ${ }^{2}$
}

\author{
* Correspondence: \\ subrata_dibru@rediffmail.com \\ ${ }^{1}$ Department of Statistics, Dibrugarh \\ University, Dibrugarh 786004, \\ Assam, India \\ Full list of author information is \\ available at the end of the article
}

\begin{abstract}
A new four parameter extended Conway-Maxwell-Poisson (ECOMP) distribution which unifies the recently proposed COM-Poisson type negative binomial (COM-NB) distribution [Chakraborty, S. and Ong, S. H. (2014): A COM-type Generalization of the Negative Binomial Distribution, Accepted in Communications in Statistics-Theory and Methods] and the generalized COM-Poisson (GCOMP) distribution [Imoto, T. :(2014) A generalized Conway-Maxwell-Poisson distribution which includes the negative binomial distribution, Applied Mathematics and Computation, 247, 824-834] is proposed. The additional parameter allows this distribution to have longer (shorter) tail compared to COM-NB and GCOMP. The proposed distribution can be formulated as an exponential combination of negative binomial and COM-Poisson distribution and also arises from a queuing system with state dependent arrival and service rates and belongs to exponential family when one of the parameter is considered as nuisance. Important distributional, reliability and stochastic ordering properties along with asymptotic approximations for the normalizing constant and the mean of this distribution is investigated. Method of parameter estimation and three comparative data fitting applications are also discussed.
\end{abstract}

Keyword: COM-Poisson, COM-Negative binomial, Generalized COM-Poisson, State dependent service and arrival rate Queues, Laplace method

Mathematics Subject Classification (2010): 62E15, 60K25, 62N05

\section{Springer}

\section{Introduction}

Recently, two new generalizations of the well known COM-Poisson (Conway and Maxwell 1962) was proposed. One by Chakraborty and Ong (2014) known as the COM-Negative binomial distribution and the other by Imoto (2014) referred to as the generalized COM-Poisson Distribution. In this section we briefly introduce these two distributions along with a hypergeometric type series which is used in the sequel.

COM-Poisson type negative binomial distribution: Chakraborty and Ong (2014) proposed a new COM-Poisson type generalization of negative binomial distribution that includes some well-known distributions including COM-Poisson, Negative Binomial (page 208-250, Chapter 5, Johnson et al. 2005), as particular case and Bernoulli (page 108, Chapter 3, Johnson et al. 2005), COM-Poisson as limiting cases 
among others. This distribution is log-concave and flexible enough to model under, equi- and over dispersed count data.

A random variable (rv) $X$ is said to follow the COM - Poisson type Negative Binomial distribution with parameters $(v, p, \alpha)[\mathrm{COM}-\mathrm{NB}(v, p, \alpha)]$ if its pmf is given by

$$
\begin{aligned}
& P(X=k)=(v)_{k} p^{k} /\left\{(k !)^{\alpha}{ }_{1} H_{a-1}(v ; 1 ; p)\right\}, k=0,1,2, \cdots \\
& \text { Where } \quad{ }_{1} H(v ; 1 ; p)=\sum_{k=0}^{\infty}(v)_{k} p^{k} /(k !)^{\alpha}
\end{aligned}
$$

The distribution is defined in the parameter space

$$
\Theta_{C O M-N B}=\{v>0, p>0, \alpha>1\} \cup\{v>0,0<p<1, \alpha=1\} .
$$

When $\alpha$ is a positive integer, ${ }_{1} H_{\alpha-1}(v ; 1 ; p)$ can be expressed as a particular case of generalized hypergeometric series ${ }_{m} F_{n}\left(a_{1}, a_{2}, \cdots, a_{m} ; b_{1}, b_{2}, \cdots, b_{m} ; z\right)=$ $\sum_{k=0}^{\infty} \frac{\left(a_{1}\right)_{k}\left(a_{2}\right)_{k} \cdots\left(a_{m}\right)_{k}}{\left(b_{1}\right)_{k}\left(b_{2}\right)_{k} \cdots\left(b_{n}\right)_{k}} \frac{z^{k}}{k !}$ as ${ }_{1} F_{\alpha-1}(v ; 1,1, \cdots, 1 ; p)$.

Generalized COM-Poisson distribution: Imoto (2014) proposed another generalization where an rv $X$ is said to follow the GCOM-Poisson distribution with parameters $(v, p, \beta)$ that is $\operatorname{GCOMP}(v, p, \beta)$ if its pmf is given by

$$
\begin{aligned}
& P(X=k)=\frac{\{\Gamma(v+k)\}^{\beta}}{C(\beta, v, p)} \frac{p^{k}}{k !} \\
& \text { Where } \quad C(\beta, v, p)=\sum_{k=0}^{\infty} \frac{\{\Gamma(v+k)\}^{\beta}}{k !} p^{k}
\end{aligned}
$$

The distribution is defined in the parameter space

$$
\Theta_{G C O M P}=\{v>0, p>0, \beta<1\} \cup\{v>0,0<p<1, \beta=1\} .
$$

A hypergeometric type series: We introduce the series

$$
{ }_{m} S_{a}^{\beta}\left(a_{1}, a_{2}, \cdots, a_{m} ; b ; p\right)=\sum_{k=0}^{\infty} \frac{\left\{\left(a_{1}\right)_{k}\right\}^{\beta}\left(a_{2}\right)_{k} \cdots\left(a_{m}\right)_{k}}{\left\{(b)_{k}\right\}^{\alpha}} \frac{p^{k}}{k !},
$$

where $(a)_{k}=a(a+1) \cdots(a+k-1)=\Gamma(a+k) / \Gamma a$ is the Pochhammer's notation (see Johnson et al. 2005, chapter 1, page 2). The series converges if (i) for any finite $p$, $\beta+m-2<\alpha$ or (ii) $|p|<1, \beta+m-2<\alpha$. For $\alpha, \beta$ and $m$ all positive integers, it reduces to a particular case of the generalized hypergeometric function ${ }_{\beta+m-1} F_{\alpha}\left(a_{1}\right.$, $\left.a_{1}, \cdots, a_{1}, a_{2}, \cdots, a_{m} ; b, b, \cdots, b ; p\right)$. With this notation we have

$$
\sum_{k=0}^{\infty}\left\{(v)_{k}\right\}^{\beta} p^{k} /(k !)^{\alpha}={ }_{1} S_{a-1}^{\beta}(v ; 1 ; p)={ }_{\beta} F_{a-1}(v ; 1,1, \cdots, 1 ; p)
$$

Some important special cases of ${ }_{1} S_{a-1}^{\beta}(v ; 1 ; p)$ are

i. ${ }_{1} S_{a-1}^{1}(v ; 1 ; p)={ }_{1} H_{a-1}(v ; 1 ; p)$ [Chakraborty and Ong, 2014]

ii. ${ }_{1} S_{0}^{\beta}(v ; 1 ; p)=C(\beta, v, p) /(\Gamma v)^{\beta}$ [Imoto 2014]

iii. $S_{0}^{1}(v ; 1 ; p)=(1-p)^{-v}$ [geometric series] 
iv. ${ }_{1} S_{a-1}^{\beta}(1 ; 1 ; p)=Z(p, \alpha-\beta)$ [Conway and Maxwell 1962]

v. ${ }_{1} S_{y}^{y}(1 ; 1 ; p)=\exp (p)$

Some important limiting cases of ${ }_{1} S_{a-1}^{\beta}(v ; 1 ; p)$ are

vi. $\lim _{\alpha \rightarrow \infty} S_{1}^{\beta} S_{a-1}(v ; 1 ; p)=1+v^{\beta} p$.

vii. $\lim _{\alpha \rightarrow \infty} S_{a-1}^{\beta}(v ; 1 ; p)=\sum_{k=0}^{\infty} \lambda^{k} /(k !)^{\alpha}=Z(\lambda, \alpha)$, where $v^{\beta} p=\lambda$ is finite positive.

In the present article we propose a natural four parameter extension of the COM-Poisson distribution which includes the recently introduced COM-NB and GCOM-Poisson distributions as special cases. This new distribution with additional parameters is more flexible in terms of tail length and dispersion index. The definition of the proposed distribution along with some of its important distributional properties are presented in the Section 2. Reliability and stochastic ordering results are discussed in Section 3. In Section 4 we presented applications of the proposed distribution by considering three real life data sets. Concluding remarks is provided in the Section 5 which if followed by an appendix containing the proofs of the results and propositions in the article.

\section{Extended COM-Poisson (ECOMP) distribution}

Here we introduce a new distribution that unifies both the COM-NB and GCOMP distributions.

Definition 1. An rv $X$ is said to follow the extended COM-Poisson distribution with parameters $(v, p, \alpha, \beta)[\operatorname{ECOMP}(v, p, \alpha, \beta)]$ iff its pmf is given by

$$
P(X=k)=\frac{\left\{(v)_{k}\right\}^{\beta}}{{ }_{1} S_{a-1}^{\beta}(v ; 1 ; p)} \frac{p^{k}}{(k !)^{\alpha}}=\frac{\{\Gamma(v+k)\}^{\beta}}{(\Gamma v)_{1}^{\beta} S_{a-1}^{\beta}(v ; 1 ; p)} \frac{p^{k}}{(k !)^{\alpha}}
$$

The distribution is defined in the parameter space

$$
\Theta_{E-C O M}=\{v \geq 0, p>0, \alpha>\beta\} \cup\{v>0,0<p<1, \alpha=\beta\} .
$$

It may be noted that unlike in the COM-NB distribution where the parameter $\alpha \geq 1$ and in the GCOMP distribution where the parameter $\beta \leq 1$, in the ECOMP distribution these two parameters can be either positive or negative with the restriction of $\alpha \geq \beta$.

Particular cases: The ECOMP $(v, p, \alpha, \beta)$ distribution reduces to COM-NB $(v, p, \alpha)$ for $\beta=1$, to $\operatorname{GCOMP}(v, p, \beta)$ for $\alpha=1$, to $\operatorname{COMP}(p, \alpha-\beta)$ for $v=1$, to $\operatorname{COMP}(p, \alpha)$ for $\beta=0$, to Poisson $(p)$ for $\nu=1, \alpha=\beta+1$, also to Poisson $(p)$ for $\beta=0, \alpha=1$, to NB $(v, p)$ for $\alpha=\beta=1$ and to a new generalization of NB(NGNB) distribution when $\alpha=\beta=\gamma$ with pmf

$$
P(X=k)=\left(\begin{array}{c}
v+k-1 \\
k
\end{array}\right)^{\gamma} p^{k} / 1 S_{y-1}^{y}(v ; 1 ; p)
$$

For $0<v \leq 1$, the distribution in (7) is log-convex as will be seen in proposition 4 in the Section 2.7. 


\subsection{Shape of the pmf}

It is observed from the plots of the pmf of the $\operatorname{ECOMP}(v, p, \alpha, \beta)$ distribution for different values of the parameters in Fig. 1, that the distribution is very flexible and can be non increasing with mode at zero, unique non zero mode, two modes and also bimodal with one mode always at zero.

\subsection{Approximations of the normalizing constant}

\subsubsection{Approximation using truncation of the series}

The normalizing constant ${ }_{1} S_{a-1}^{\beta}(v ; 1 ; p)$ of the $\operatorname{ECOMP}(v, p, \alpha, \beta)$ distribution is not expressed in a closed form and includes the summation of infinite series. Therefore, we

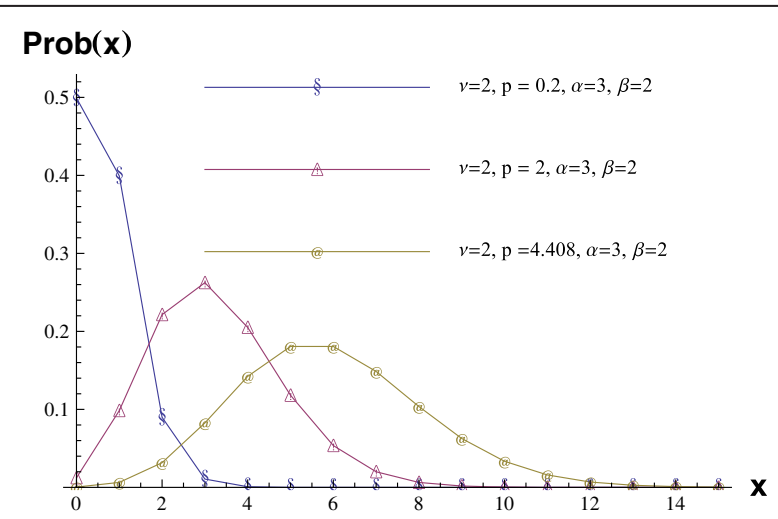

\section{$\operatorname{Prob}(\mathbf{x})$}

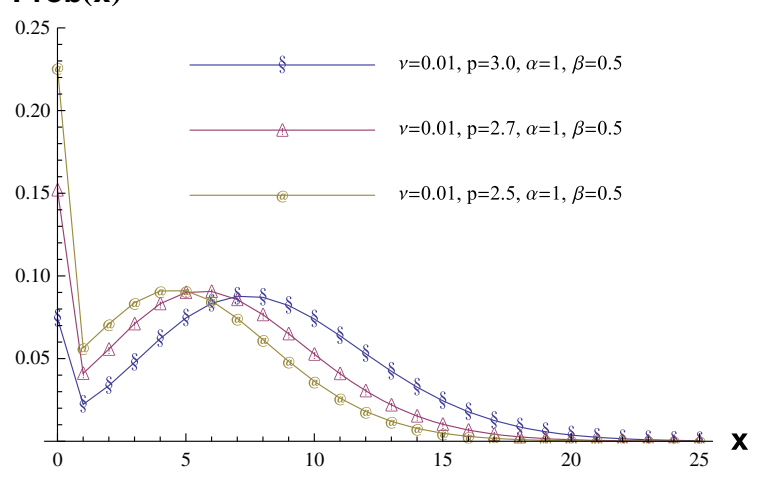

\section{$\operatorname{Prob}(\mathbf{x})$}

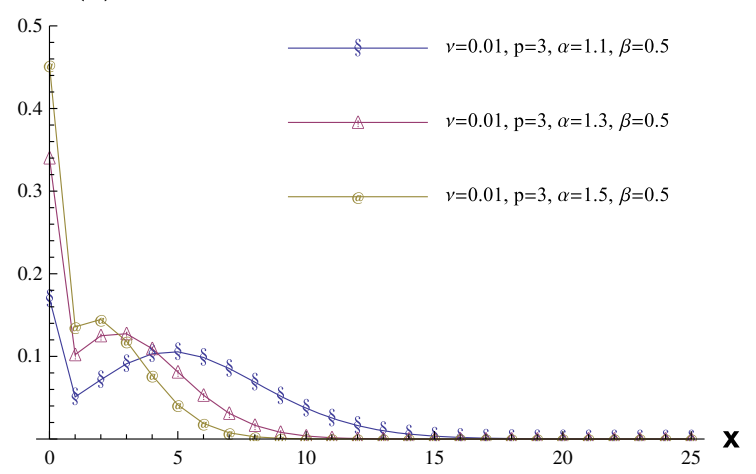

Fig. 1 pmfs of ECOMP 
need approximations of this constant to compute the pmf and moments of the distribution numerically.

A simple approximation is to truncate the series, that is

$$
{ }_{1} S_{a-1, m}^{\beta}(v ; 1 ; p)=\sum_{k=0}^{m} \frac{\left\{(v)_{k}\right\}^{\beta}}{(k !)^{\alpha}} p^{k},
$$

where $m$ is an integer chosen such that $\varepsilon_{m}=(v-m+1)^{\beta} p / m^{\alpha}<1$. The relative truncation error is then given by the expression $R_{m}(v, p, \alpha, \beta)=$ $\left\{{ }_{1} S_{a-1}^{\beta}(v ; 1 ; p)-{ }_{1} S_{a-1, m}^{\beta}(v ; 1 ; p)\right\} /{ }_{1} S_{a-1, m}^{\beta}(v ; 1 ; p)$. Then the relative error about the pmf is give by $\left\{P_{m}(k)-P(k)\right\} / P(k)$, where $P(k)$ is given by the right hand side (r.h.s.) of equation (6) in Section 2 and $P_{m}(k)$ is given by the r.h.s. of (6) with ${ }_{1} S_{a-1}^{\beta}(v ; 1 ; p)$ substituted by ${ }_{1} S_{a-1, m}^{\beta}(v ; 1 ; p)$. The upper bound of the relative truncation error is then found to be

$$
R_{m}(v, p, \alpha, \beta)<\frac{\left\{(v)_{m+1}\right\}^{\beta} p^{m+1}}{\{(m+1) !\}^{\alpha}{ }_{1} S_{a-1, m}^{\beta}(v ; 1 ; p)} \sum_{k=0}^{\infty} \varepsilon_{m}^{k}=\frac{\left\{(v)_{m+1}\right\}^{\beta} p^{m+1}}{\left\{\left(1-\varepsilon_{m}\right)(m+1) !\right\}^{\alpha}{ }_{1} S_{a-1, m}^{\beta}(v ; 1 ; p)}
$$

For $\alpha-\beta \geq 1$, this truncated approximation is good because $\varepsilon_{m}=O(1 / m)$ and thus, the truncation point $m$ is not large. However, for $0<\alpha-\beta<1$ and $p>1$, the truncation point become too large to compute the approximation. For example, when $v=1.5, p=3, \alpha=3.1, \beta=3, m$ has to be over 50,000 . This is not practicable. To avoid this difficulty it is useful to make a restriction for the parameter $p$ such that $p<1$ when $\alpha-\beta \rightarrow 0$. For example, with the restriction $p<10^{\alpha-\beta}$, we see the relative truncation error $R_{50}(1.5,3,3.1,3)<0.001$.

\subsubsection{Asymptotic approximation of the normalizing constant using the Laplace's method}

It is also useful to consider an asymptotic approximation formula of the normalizing constant ${ }_{1} S_{a-1}^{\beta}(\nu, 1, p)$. The approximation formula by the Laplace's method (Bleistein and Handelsman 1986, Ch 8.3, pages 331-340) is given by

$$
{ }_{1} S_{a}^{\beta}(v ; 1, p) \approx \frac{p^{\{1-\alpha+(2 v-1) \beta\} / 2(\alpha-\beta)} \exp \left\{(\alpha-\beta) p^{1 /(\alpha-\beta)}\right\}}{(2 \pi)^{(\alpha-\beta-1) / 2} \sqrt{\alpha-\beta}\{\Gamma(\nu)\}^{\beta}}
$$

This formula reduces to the asymptotic formula by Minka et al. (2003) when $v=1$ or $\beta=0$ and that by Imoto (2014) when $\alpha=1$. The proof and numerical investigation about the formula (9) are given in Appendix A.1.

\subsection{Recurrence relation for probabilities}

The ECOMP $(v, p, \alpha, \beta)$ pmf has a simple recurrence relation given by

$$
\frac{P(X=k+1)}{P(X=k)}=\frac{p(v+k)^{\beta}}{(k+1)^{\alpha}} \Rightarrow(k+1)^{\alpha} P(X=k+1)=p(v+k)^{\beta} P(X=k)
$$

with $P(X=0)=\left[{ }_{1} S_{a-1}^{\beta}(v ; 1 ; p)\right]^{-1}$. This will be useful for the computation of the probabilities. Further using (10) we can see that the $\operatorname{ECOMP}(v, p, \alpha, \beta)$ distribution has a longer (shorter) tail than the $\mathrm{COM}-\mathrm{NB}(v, p, \alpha)$ for $\alpha<(>) 1$ and a longer (shorter) tail than the $\operatorname{GCOMP}(v, p, \beta)$ for $\beta>(<) 1$. 


\subsection{Exponential family}

The pmf in (6) can also be expressed as

$$
P(X=k)=\exp \left[\beta \log (v)_{k}-\beta \log \Gamma(v)-\alpha \log k !+k \log p-\log _{1} S_{a-1}^{\beta}(v ; 1 ; p)\right]
$$

Which immediately implies that the ECOMP $(v, p, \alpha, \beta)$ distribution belongs to the exponential family with parameters $(\log p, \alpha, \beta)$ when $v$, is a nuisance parameter or when its value is given.

\subsection{Index of dispersion}

The pmf of ECOMP $(v, p, \alpha, \beta)$ distribution in (6) can be seen as a weighted Poisson $(p)$ distribution with weight function $w(x)=\{\Gamma(v+x)\}^{\beta} /(\Gamma(1+x))^{\alpha-1}$. As such it will be over (under) dispersed if $w(x)$ in log-convex (log-concave). That is if $\frac{d^{2}}{d x^{2}} \log [w(x)] \geq(\leq) 0$. [See theorem 4 of Kokonendji et al. 2008]

$$
\begin{aligned}
& \Rightarrow \beta \frac{d^{2}}{d x^{2}} \log \Gamma(v+x)+(1-\alpha) \frac{d^{2}}{d x^{2}} \log \Gamma(1+x) \geq(\leq) 0 \\
& \Rightarrow \beta \sum_{k \geq 0} \frac{1}{(v+x+k)^{2}}-(\alpha-1) \sum_{k \geq 0} \frac{1}{(x+1+k)^{2}} \geq(\leq) 0
\end{aligned}
$$

[On using result 6.4.10 page 260 from Abramowitz and Stegun, 1970].

Hence, ECOMP $(v, p, \alpha, \beta)$ is over dispersed (i) if $\alpha<1, \beta \geq 0$ for all $v$ (ii) if $\{\alpha \geq 1, \beta>0\}$ or $\{\alpha<1, \beta<0\}$ when $\{0<v \leq 1, \beta \leq \alpha \leq \beta+1\}$ or $\{v>1, \alpha \leq 1\}$ and under dispersed (i) if $\alpha \geq 1, \beta<0$ for all $v$ (ii) for $\{\alpha \geq 1, \beta>0\}$ or $\{\alpha<1, \beta<0\}$ if $\{0<v \leq 1, \alpha \geq \beta+1\}$ or $\{v>1, \alpha \geq 1\}$.

As a particular cases of the above result, when $\beta=1$, we can see that the COM$\mathrm{NB}(v, p, \alpha)$ distribution always over dispersed for $\{0<v \leq 1,1 \leq \alpha<2\}$ or $\{v>1, \alpha=$ $1\}$ and under dispersed compared to COMP distribution for $\{0<v \leq 1, \alpha \geq 2\}$. Similarly when $\alpha=1$, the $\operatorname{GCOMP}(v, p, \beta)$ distribution is seen to be is over dispersed for $0<\beta \leq 1$ and under dispersed for $\beta<0$. When $v=1$, we derive that COMP $(p, \alpha-\beta)$ is over dispersed for $\alpha-\beta>1$ under dispersed for $\alpha-\beta<1$ and equidispersed when $\alpha-\beta=1$. Finally, the new generalized NB distribution with pmf (7) is over dispersed when $\gamma=1$ (which is when it reduces to Negative binomial) and under dispersed if $\gamma>1$.

It can also be checked that ECOMP $(v, p, \alpha, \beta)$ is over (under) dispersed for $\alpha \geq \beta>(\leq) 0$ w.r.t. COM-NB $(v, p, \alpha)$ and w.r.t. GCOM-Poisson $(v, p, \beta)$ it is over (under) dispersed for $\beta \leq \alpha<1 \quad(1<\beta \leq \alpha)$.

\subsection{Different formulations of ECOMP $(v, p, a, \beta)$}

Two different formulations of the proposed distribution are presented in this section.

\subsubsection{ECOMP $(v, p, a, \beta)$ as a distribution from a queuing set up}

Like the COM-Poisson distribution, the $\operatorname{ECOMP}(v, p, \alpha, \beta)$ distribution can also be derived as the probability of the system being in the $k^{\text {th }}$ state for a queuing system with state dependent service and arrival rate.

Consider a single server queuing system with state dependent (that is dependent on the system state, $k^{\text {th }}$ state means $k$ number of units in the system) arrival rate $\lambda_{k}=(v+k)^{\beta} \lambda$, and state dependent service rate $\mu_{k}=k^{\alpha} \mu$, where, $1 / \mu$ and $1 / \lambda$ are 
respectively the normal mean service and mean arrival time for a unit when that unit is the only one in the system; $\alpha$ and $v$ are the pressure coefficients, reflecting the degree to which the service and arrival rates of the system are affected by the system state.

Proposition 1. Under the above set up where the arrival rate and the service rate increases exponentially as queue lengthens (i.e. as $k$ increases) the probability of the system being in the $k^{\text {th }}$ state is $\operatorname{ECOMP}(v, p, \alpha, \beta)$.

Proof: See Appendix B.1

\subsubsection{ECOMP $(v, p, a, \beta)$ as exponential combination formulation}

The general form of the exponential combination of two pmfs say $f_{1}\left(x ; \theta_{1}\right)$ and $f_{2}\left(x ; \theta_{2}\right)$ is given by (Atkinson 1970)

$$
\left\{f_{1}\left(x ; \theta_{1}\right)\right\}^{\beta}\left\{f_{2}\left(x ; \theta_{2}\right)\right\}^{1-\beta} / \sum f_{1}\left(x ; \theta_{1}\right)^{\beta} f_{2}\left(x ; \theta_{2}\right)^{1-\beta}
$$

This combining of the pmf was suggested by Cox $(1961,1962)$ for combining the two hypotheses $\left(\beta=1\right.$, i.e. the distribution is $f_{1}$ and $\beta=0$ that is the distribution is $f_{2}$ ) in a general model of which they would both be special cases. The inferences about $\beta$ made in the usual way and testing the hypothesis that the value of $\beta$ is zero or one is equivalent to testing for departures from one model in the direction of the other.

Proposition 2. ECOMP $(v, p, \alpha, \beta)$ distribution is an exponential combination NB $(v, \lambda)$ and COM-Poisson $(\mu, \theta)$ distributions, with $\lambda^{\beta} \mu^{1-\beta}=p$ and $\alpha=\theta(1-\beta)+\beta$.

Proof: See Appendix B.2.

From the above formulations it is clear that for $\operatorname{ECOMP}(\nu, p, \alpha, \beta), \beta$ close to zero will indicate departure from COM-Poisson towards $\mathrm{NB}$, while $\beta$ close to one will indicate the reverse. Thus ECOMP $(v, p, \alpha, \beta)$ can also be regarded as a natural extension of COM-Poisson, and negative binomial distributions.

\subsection{Log-concavity and modality}

Proposition 3. The ECOMP $(v, p, \alpha, \beta)$ has a $\log$-concave $p m f$ when $\{v>1, p>0, \alpha \geq \beta\}$

Proof: See Appendix B.3.

From the above result the corresponding results of COM-NB $(v, p, \alpha)$ and GCOMP $(v, p, \beta)$ can be obtained as particular cases. That is COM-NB $(v, p, \alpha)$ is log-concave when $\{v>1, p>0, \alpha \geq 1\}$ and $\operatorname{GCOMP}(v, p, \beta)$ is log-concave when $\{v>1, p>0, \beta \leq 1\}$.

Following two important results follows as a consequence of log-concavity:

If $\{v \geq 1, p>0, \alpha>\beta\}$ the ECOMP $(v, p, \alpha, \beta)$ distribution is

$>$ a strongly unimodal distribution

$>$ has an increasing failure rate function

Using the recurrence relation of the probabilities in (10) it is observed that the $\operatorname{ECOMP}(v, p, \alpha, \beta)$ has

(i) a non increasing pmf with a unique mode at $X=0$ if $v^{\beta} p<1$, e.g. $v=2, \alpha=3, \beta=2, p$ should be less than 0.25 to have unique mode at $X=0$.

(ii) a unique mode at $X=k$ if $k^{\alpha} /(v+k-1)^{\beta}<p<(k+1)^{\alpha} /(v+k)^{\beta}$ 
e.g. $v=2, \alpha=3, \beta=2, p$ should be between 1.6875 and 2.560 to have unique mode at $X=3$.

(iii) two modes at $X=k$ and $X=k-1$ if $(v+k-1)^{\beta} p=k^{\alpha}$. In particular the two modes are at $X=0$ and $X=1$ if $v^{\beta} p=1$.

e.g. $v=2, \alpha=3, \beta=2, p$ should be equal to 4.408 to have two modes at $X=5$ and $X=6$.

Graphical illustrations of the above three examples are presented in the first plots of Fig. 1. It is interesting to note that the distribution may be bimodal with one of the mode always at zero as shown the last two plots in Fig. 1.

Proposition 4. ECOMP $(v, p, \alpha, \beta)$ has a log-convex pmf for $\{0<v \leq 1, \alpha=\beta\}$

Proof. See Appendix B.4.

Following important results follows as a consequence of log-convexity:

If $\{v \leq 1, p>0, \alpha=\beta\}$ the ECOMP $(v, p, \alpha, \beta)$ distribution with pmf in (7)

$>$ is Infinitely divisible (see Warde and Katti 1971) distribution, hence Discrete

Compound Poisson distribution. (see page 409 of Gómez-Déniz et al. 2011)

$>$ has an decreasing failure rate function, hence increasing mean residual life function

$>$ has an upper bound for variance as $p v^{\beta}$ (using result of page 410 of Gómez-Déniz et al. 2011)

\subsection{Moments}

The $r^{\text {th }}$ factorial moment $E\left(X^{[r]}\right)=\mu^{[r]}$ of the $\operatorname{ECOMP}(\nu, p, \alpha, \beta)$ is given by

$$
\begin{aligned}
& \mu^{[r]}=\frac{\left\{(v)_{r}\right\}^{\beta} p^{r}}{(r !)^{\alpha-1}} \frac{S_{a-1}^{\beta}(v+r, r+1, p)}{{ }_{1} S_{a-1}^{\beta}(v, 1, p)} \\
& =\frac{\left\{(v)_{r}\right\}^{\beta} p^{r}{ }_{\beta} F_{a-1}(v+r ; r+1, r+1, \cdots, r+1 ; p)}{(r !)^{\alpha-1}},
\end{aligned}
$$

where the second expression in terms of hypergeometric function is for the case when $\alpha, \beta$ are both positive integers.

Since the $\operatorname{ECOMP}(\nu, p, \alpha, \beta)$ distribution is a member of exponential family (see Section 2.4), the mean is given by differentiating the logarithm of the normalizing constant with respect to $p$. Hence an asymptotic approximation for the mean is obtained by differentiating the logarithm of the function (9) as

$$
p^{1 /(\alpha-\beta)}+\frac{1-\alpha+(2 v-1) \beta}{2(\alpha-\beta)} .
$$

This function approximates the mean of the $\operatorname{ECOMP}(v, p, \alpha, \beta)$ distribution for large $p$ and small $|\alpha-\beta|$, where it is difficult to compute the approximation by truncation. A numerical illustration of this asymptotic approximation is presented in the Appendix A.2. 


\section{Reliability characteristics and stochastic ordering}

\subsection{Survival and failure rate functions}

The survival function is given by

$$
\begin{aligned}
S(t)=1-P(X<t) & =1-\frac{1}{{ }_{1} S_{a-1}^{\beta}(v, ; 1 ; p)} \sum_{k=0}^{t-1} \frac{\left\{(v)_{k}\right\}^{\beta} p^{k}}{(k !)^{\alpha}} \\
& =1-\frac{1}{{ }_{\beta} F_{a-1}(v ; 1,1, \cdots, 1 ; p)} \sum_{k=0}^{t-1} \frac{\left\{(v)_{k}\right\}^{\beta} p^{k}}{(k !)^{\alpha}} .
\end{aligned}
$$

Alternatively, $S(t)$ can also be expressed as

$$
\begin{aligned}
S(t) & =\frac{(v)_{t} p^{t}}{(t !)^{\alpha}} \frac{{ }_{2} S_{a-1}^{\beta}(v+t, 1 ; t+1 ; p)}{{ }_{1} S_{a-1}^{\beta}(v, ; 1 ; p)} \\
& =\frac{(v)_{t} p^{t}}{(t !)^{\alpha}} \frac{\beta_{+1} F_{a-1}(v+t, 1 ; t+1, t+1, \cdots, t+1 ; p)}{{ }_{\beta} F_{a-1}(v ; 1,1, \cdots, 1 ; p)} .
\end{aligned}
$$

The failure rate function is given by

$$
r(t)=\frac{P(X=t)}{P(X \geq t)}=\frac{1}{{ }_{2} S_{a-1}^{\beta}(v+t, 1 ; t+1 ; p)}=\frac{1}{\beta+{ }_{1} F_{a}(v+t, 1 ; t+1, t+1, \cdots, t+1 ; p),}
$$

where the second expression in terms of hypergeometric function is for the case when $\alpha, \beta$ are positive integers.

\subsection{Stochastic orderings}

An $\operatorname{rv} X$ with pmf $P(X=n)$ is said to be smaller than another $\operatorname{rv} Y \operatorname{pmf} P(Y=n)$ in the likelihood ratio order that is $X \leq{ }_{l r} Y$ if $P(Y=n) / P(X=n)$ increases in $n$ over the union of the supports of $X$ and $Y$. Again $X \leq{ }_{l r} Y$ implies $X$ is smaller than $Y$ in the hazard rate order and subsequently in the mean residual (MRL) life order (see Gupta et al. 2014).

Theorem 1. $X \sim \operatorname{ECOMP}(v, p, \alpha, \beta)$ is smaller than $Y \sim \operatorname{COM-NB}(v, p, \alpha)$ in the likelihood ratio order i.e. $X \leq{ }_{l r} Y$ when $\beta<1$.

Proof: If $X \sim \operatorname{ECOMP}(v, p, \alpha, \beta)$ and $Y \sim \operatorname{COM}-\mathrm{NB}(v, p, \alpha)$, then

$$
\frac{P(Y=n)}{P(X=n)}=\left\{(v)_{n}\right\}^{1-\beta} \frac{S_{a-1}^{\beta}(v, 1, p)}{{ }_{1} S_{a-1}^{1}(v, 1, p)} .
$$

This is clearly increasing in $n$ as $\beta<1$ (Definition 1.C.1 of Chapter 1, Shaked and Shanthikumar 2007 and Gupta et al. 2014). Hence the result is proved.

As an implication of theorem 1, we get $X \leq_{h r} Y \Rightarrow X \leq_{M R L} Y$, for $\beta<1$.

Theorem 2. $X \sim \operatorname{ECOMP}(v, p, \alpha, \beta)$ is smaller than $Y \sim \operatorname{GCOMP}(v, p, \beta)$ in the likelihood ratio order i.e. $X \leq_{l r} Y$ when $\alpha>1$.

Proof: If $X \sim \mathrm{ECOM}-\mathrm{NB}(v, p, \alpha, \beta)$ and $Y \sim \operatorname{GCOMP}(v, p, \beta)$, then

$$
\frac{P(Y=n)}{P(X=n)}=\frac{(n !)^{\alpha-1}}{(\Gamma v)^{\beta}} \frac{{ }_{1} S_{a-1}^{\beta}(v, 1, p)}{{ }_{1} S_{0}^{\beta}(v, 1, p)} .
$$

This is clearly increasing in $n$ as $\alpha>1$ (Definition 1.C.1 of Chapter 1, Shaked and Shanthikumar 2007 and Gupta et al. 2014). Hence the result is proved. 
As an implication of theorem 2, we get $X \leq_{h r} Y \Rightarrow X \leq_{M R L} Y$, for $\alpha>1$.

\section{Numerical examples}

To fit the proposed distribution, we have to estimate the parameters $(v, p, \alpha, \beta)$ in (6). The maximum likelihood (ML) estimation is often used for fitting to real data, but the $\log$ likelihood function of the proposed distribution

$$
\mathrm{L}(v, p, \alpha, \beta)=\beta \sum_{i=0}^{k} f_{i} \log (v)_{i}-\alpha \sum_{i=0}^{k} f_{i} \log i !+\log p \sum_{i=0}^{k} i f_{i}-N \log _{1} S_{a-1}^{\beta}(v, 1, p)
$$

where $f_{i}$ is the observed frequency of $i^{\text {th }}$ observed value(event), $N=\sum_{i=1}^{k} f_{i}, k$ is the highest observed value, has some local maximum points for some datasets, or the likelihood equations do not always have unique solution. Therefore, we use the profile likelihood estimation. We first consider the maximum likelihood estimation by fixing the parameter $v$ and finding the maximum point $\left(\hat{p}_{v}, \hat{\alpha}_{v}, \hat{\beta}_{v}\right)$ of the function (13). The maximum point $\left(\hat{p}_{v}, \hat{\alpha}_{v}, \hat{\beta}_{v}\right)$ is uniquely determined because the proposed distribution belongs to the exponential family when $v$ is fixed. For finding $\left(\hat{p}_{v}, \hat{\alpha}_{v}, \hat{\beta}_{v}\right)$ computationally, it is convenient to use some initial values. The simple initial values can be obtained as follow. Putting $c_{x}=P(X=x+1) / P(X=x)$ and $d_{x}=\log \left(c_{x+1} / c_{x}\right)$, where $X$ is the rv following $\operatorname{ECOMP}(v, p, \alpha, \beta)$ distribution, we have the equation

$$
A_{x}(v)\left(\begin{array}{c}
\alpha \\
\beta
\end{array}\right)=\left(\begin{array}{c}
d_{x} \\
d_{x+1}
\end{array}\right) \text {, where } A_{x}(v)=\left(\begin{array}{cc}
\log \frac{x+1}{x+2} & \log \frac{v+x+1}{v+x} \\
\log \frac{x+2}{x+3} & \log \frac{v+x+2}{v+x+1}
\end{array}\right) .
$$

For given $v$, we choose the integer $k$ such that $\left|A_{k}(v)\right| \neq 0$ and put

$$
\left(\begin{array}{c}
s_{1, k}(v) \\
s_{2, k}(v)
\end{array}\right)=A_{k}(v)^{-1}\left(\begin{array}{c}
d_{k} \\
d_{k+1}
\end{array}\right)
$$

where $P(X=x)$ is substituted with $f_{x}$ in $d_{x}$. Then we can obtain the initial values $\left(\tilde{p}_{k}(v), \widetilde{\alpha}_{k}(v), \widetilde{\beta}_{k}(v)\right)$ for $(p, \alpha, \beta)$ as

$$
\tilde{\alpha}_{k}(v)=\left\{\begin{array}{cc}
s_{1, k}(v) & s_{1, k}(v)>s_{2, k}(v) \\
s_{2, k}(v) & \text { otherwise }
\end{array}, \widetilde{\beta}_{k}(v)=s_{2, k}(v) \text { and } \tilde{p}_{l}(v)=\frac{(l+1) \widetilde{\alpha_{k}(v)}}{(v+l) \widetilde{\beta_{k}}(v)},\right.
$$

where $l$ is the lowest observed value (e.g. $l=0$ for neither censored nor truncated data). These values are available even for the truncated version of $\operatorname{ECOMP}(v, p, \alpha, \beta)$ distribution. Then by studying the behavior of $\mathrm{L}\left(v, \hat{p}_{v}, \hat{\alpha}_{v}, \hat{\beta}_{v}\right)$ with $v$ varying, we find the range of $v$ where the function will give the global maximum. For the range, the maximum point of the function (13) gives the ML estimates $(\hat{v}, \hat{p}, \hat{\alpha}, \hat{\beta})$.

By using this method, we fit the proposed distribution to three datasets and compare with NB $(r, p)$, COMP $(\theta, p)$ COM-NB $(v, p, \alpha)$ and GCOMP $(v, p, \beta)$. Simultaneously, we fit Delaporte distribution, which is derived from the convolution of a NB $(r, p)$ and Poisson $(\lambda) \mathrm{rv}$, and some mixed Poisson distributions; mixing with generalized gamma 
distribution of Agarwal and Kalla (1996) with parameters $(\delta, m, \alpha, n)$, mixing with generalized inverse Gaussian gamma distribution of Jorgensen (1982) with parameters $(\chi, \eta, \omega, \lambda)$, mixing with generalized exponential distribution of Ong and Lee (1986) with parameters $(v, a, d, \beta)$. These distributions are derived as the generalized negative binomial distributions and used for long-tailed count data. The detailed studies are given in Gupta and Ong (2005). Here we show only the best fitting distribution among these distributions in Gupta and Ong (2005). The performances of various distributions are compared using the $\chi^{2}$ goodness of fit and the Akaike Information Criterion (AIC). Following Burnham and Anderson (2004) we look at the difference $\Delta_{i}=\mathrm{AIC}_{i}-\mathrm{AIC}_{\mathrm{min}}$ where $\mathrm{AIC}_{\min }$ is the minimum of the $\mathrm{AIC}$ values of the all the fitted model and $\mathrm{AIC}_{i}$ is that of the $i^{\text {th }}$ model. According to Burnham and Anderson (2004), models having $\Delta_{i} \leq 2$ had substantial support (evidence) and those in which $4 \leq \Delta_{i}$ have considerably less support. For computing the $\chi^{2}$ goodness of fit statistics we group the cells whose expected number is less than 5 such that the expected number of grouped cell is not less than 5 .

\subsection{The spots in southern pine beetle}

The first example is the frequency distribution of Corbet's Malayan Buttery with zeros (Corbet 1942). Corbet caught altogether 620 species, but he also estimated that the total buttery fauna of the area contained 924 species, so that 304 species were missing from the collection and treated as count zero. In this dataset, the counts more than 24 are grouped as $25+$, so we use the log-likelihood function of the form

$$
\sum_{i=0}^{24} f_{i} \log P(X=i)+f_{25} \log P(X \geq 25)
$$

where $X$ is the rv of the fitted distribution.

Comparing the performance of the distributions presented in Table 1, we see that the Delaporte distribution gives best and marginally better fit than the ECOMP distribution in terms of AIC and $\chi^{2}$ goodness of fit but looking at the value $\Delta_{i}$ suggests that the ECOMP distribution also has substantial support (evidence) for the data. Both theses two distributions give much better fittings for the count 0,1 and the tail part 25+ compared to the rest.

More over for it can be observed, the ML estimate $\hat{\alpha}$ of the COM-NB distribution and ML estimate $\hat{\beta}$ of the GCOMP distribution show these two distributions reduce to the negative binomial distribution, while the proposed ECOMP distribution does not seem to reduce to the negative binomial distribution. Actually, the likelihood ratio test for $H_{0}$ : Negative binomial distribution $(\alpha=\beta=1)$ Vs $H_{1}$ : ECOMP distribution $(\alpha \neq 1$ or $\beta \neq 1$ ) rejects the negative binomial distribution ( $p$-value is 0.001 ). So the ECOMP distribution brings in substantial improvement in fitting this data set over both COMNB and GCOMP distributions.

\subsection{The spots in southern pine beetle}

The second example is the frequency data of the number of spots $(k)$ in southern pine beetle, Dentroctonus frontails Zimmerman, (Coleopetra: Scolytidae), in Southeast Texas (Lin 1985). Table 2 shows the fitting results and Poi-GE means the mixed Poisson distribution with generalized exponential distribution. From $\chi^{2}$ goodness of fit 
Table 1 Distribution of Corbet's Malayan Buttery with zeros (Corbet 1942)

\begin{tabular}{|c|c|c|c|c|c|c|c|}
\hline Count & Observed & NB & Delaporte & COMP & COMNB & GCOMP & ECOMP \\
\hline 0 & 304 & 315.36 & 303.10 & 104.93 & 315.36 & 315.36 & 304.97 \\
\hline 1 & 118 & 94.24 & 123.28 & 93.03 & 94.24 & 94.24 & 117.12 \\
\hline 2 & 74 & 59.76 & 62.83 & 82.47 & 59.76 & 59.76 & 67.25 \\
\hline 3 & 44 & 44.58 & 43.29 & 73.11 & 44.58 & 44.58 & 45.92 \\
\hline 4 & 24 & 35.74 & 33.73 & 64.81 & 35.74 & 35.74 & 34.51 \\
\hline 5 & 29 & 29.85 & 27.77 & 57.45 & 29.85 & 29.85 & 27.57 \\
\hline 6 & 22 & 25.60 & 23.61 & 50.93 & 25.60 & 25.60 & 22.94 \\
\hline 7 & 20 & 22.37 & 10.51 & 45.14 & 22.37 & 22.37 & 19.66 \\
\hline 8 & 19 & 19.81 & 18.10 & 40.02 & 19.81 & 19.81 & 17.23 \\
\hline 9 & 20 & 17.73 & 16.16 & 35.47 & 17.73 & 17.73 & 15.35 \\
\hline 10 & 15 & 16.0 & 14.57 & 31.44 & 16.00 & 16.00 & 13.86 \\
\hline 11 & 12 & 14.53 & 13.23 & 27.87 & 14.53 & 14.53 & 12.64 \\
\hline 12 & 14 & 13.27 & 12.09 & 24.70 & 13.27 & 13.27 & 11.63 \\
\hline 13 & 6 & 12.18 & 11.10 & 21.90 & 12.18 & 12.18 & 10.77 \\
\hline 14 & 12 & 11.23 & 10.24 & 19.41 & 11.23 & 11.23 & 10.04 \\
\hline 15 & 6 & 10.38 & 9.49 & 17.20 & 10.38 & 10.38 & 9.39 \\
\hline 16 & 9 & 9.63 & 8.82 & 15.25 & 9.63 & 9.63 & 8.83 \\
\hline 17 & 9 & 8.96 & 8.21 & 13.51 & 8.96 & 8.96 & 8.32 \\
\hline 18 & 6 & 8.35 & 7.68 & 11.98 & 8.35 & 8.35 & 7.86 \\
\hline 19 & 10 & 7.80 & 7.19 & 10.62 & 7.80 & 7.80 & 7.45 \\
\hline 20 & 10 & 7.30 & 6.74 & 9.41 & 7.30 & 7.30 & 7.07 \\
\hline 21 & 11 & 6.84 & 6.34 & 8.34 & 6.84 & 6.84 & 6.73 \\
\hline 22 & 5 & 6.42 & 5.97 & 7.39 & 6.42 & 6.42 & 6.40 \\
\hline 23 & 3 & 6.04 & 5.63 & 6.55 & 6.04 & 6.04 & 6.10 \\
\hline 24 & 3 & 5.69 & 5.32 & 5.81 & 5.69 & 5.69 & 5.82 \\
\hline $25+$ & 119 & 114.36 & 119.00 & 45.27 & 114.36 & 114.36 & 118.57 \\
\hline Total & 924 & 924 & 924 & 924 & 924 & 924 & 924 \\
\hline $\mathrm{AlC}$ & & 4516.22 & 4508.40 & 45021.16 & 4518.22 & 4518.22 & 4510.02 \\
\hline$x^{2}$ & & 28.50 & 19.46 & 660.91 & 28.50 & 28.50 & 18.57 \\
\hline$p$-value & & 0.20 & 0.62 & 0.00 & 0.15 & 0.15 & 0.61 \\
\hline \multirow[t]{4}{*}{ MLES } & & $\hat{v}=0.31$ & $\hat{r}=0.26$ & - & $\hat{v}=0.31$ & $\hat{v}=0.31$ & $\hat{v}=2.86$ \\
\hline & & $\hat{p}=0.97$ & $\hat{p}=0.97$ & $\hat{p}=0.01$ & $\hat{p}=0.97$ & $\hat{p}=0.97$ & $\hat{p}=1.26$ \\
\hline & & & $\hat{\lambda}=0.15$ & $\hat{a}=0.89$ & $\hat{a}=1.00$ & - & $\hat{a}=-1.07$ \\
\hline & & & & & & $\hat{\beta}=1.00$ & $\hat{\beta}=-1.13$ \\
\hline
\end{tabular}

and AIC, the GCOMP distribution gives the best fitting among fitted distributions. However, a look at the value $\Delta_{i}$ suggests that the ECOMP distribution gives equally good fitting to the data. From the estimated parameters of the ECOMP distribution, we can see that fitted ECOMP distribution reduces to the new generalization of NB distribution given in equation (7) with estimated parameters $\hat{v}=0.002, \hat{p}=0.69, \hat{\gamma}=0.28$. Further, by virtue of proposition 2 in Section 2.6 .2 and we can conclude that fitted ECOMP distribution reduces to an exponential combination of NB $(0.003, \lambda)$ and Geometric $(\mu)$ in the ratio $0.28: 0.72$, where $\lambda$ and $\mu$ can be calculated using the formula given in the Section 2.6.2. 
Table 2 The number of spots in southern pine beetle (Lin 1985)

\begin{tabular}{|c|c|c|c|c|c|c|c|}
\hline Count & Observed & NB & Poi-GE & COMP & COMNB & GCOMP & ECOMP \\
\hline 0 & 1169 & 1165.32 & 1168.98 & 922.62 & 1168.56 & 1168.71 & 1169.00 \\
\hline 1 & 144 & 169.40 & 148.01 & 373.44 & 152.87 & 151.88 & 147.59 \\
\hline 2 & 92 & 78.91 & 82.91 & 151.15 & 80.17 & 80.69 & 84.21 \\
\hline 3 & 54 & 45.18 & 51.87 & 61.18 & 49.76 & 50.13 & 52.00 \\
\hline 4 & 29 & 28.28 & 33.51 & 24.76 & 32.60 & 32.75 & 33.17 \\
\hline 5 & 18 & 18.61 & 21.93 & 10.02 & 21.80 & 21.82 & 21.54 \\
\hline 6 & 10 & 12.65 & 14.44 & 4.06 & 14.68 & 14.64 & 14.14 \\
\hline 7 & 12 & 8.78 & 9.55 & 1.64 & 9.89 & 9.84 & 9.36 \\
\hline 8 & 6 & 6.20 & 6.32 & 0.66 & 6.65 & 6.60 & 6.23 \\
\hline 9 & 9 & 4.44 & 4.19 & 0.27 & 4.45 & 4.41 & 4.16 \\
\hline 10 & 3 & 3.20 & 2.78 & 0.11 & 2.96 & 2.93 & 2.79 \\
\hline 11 & 2 & 2.33 & 1.85 & 0.04 & 1.96 & 1.94 & 1.88 \\
\hline 12 & 0 & 1.71 & 1.23 & 0.02 & 1.29 & 1.28 & 1.27 \\
\hline 13 & 0 & 1.26 & 0.81 & 0.01 & 0.84 & 0.84 & 0.85 \\
\hline 14 & 1 & 0.93 & 0.54 & 0.00 & 0.55 & 0.55 & 0.58 \\
\hline 15 & 0 & 0.69 & 0.36 & 0.00 & 0.35 & 0.35 & 0.39 \\
\hline 16 & 0 & 0.52 & 0.24 & 0.00 & 0.23 & 0.23 & 0.27 \\
\hline 17 & 0 & 0.39 & 0.16 & 0.00 & 0.15 & 0.15 & 0.18 \\
\hline 18 & 0 & 0.29 & 0.11 & 0.00 & 0.09 & 0.09 & 0.12 \\
\hline 19 & 1 & 0.92 & 0.22 & 0.00 & 0.15 & 0.16 & 0.28 \\
\hline Total & 1550 & 1550.00 & 1550.00 & 1550.00 & 1550.00 & 1550.00 & 1550.00 \\
\hline AIC & & 3117.28 & 3116.33 & 3518.85 & 3113.24 & 3112.99 & 3113.84 \\
\hline$x^{2}$ & & 14.79 & 8.56 & 669.08 & 8.99 & 8.65 & 8.18 \\
\hline$p$-value & & 0.06 & 0.13 & 0.00 & 0.25 & 0.28 & 0.23 \\
\hline \multirow[t]{5}{*}{ MLES } & & $\widehat{r}=0.18$ & $\widehat{V}=0.08$ & $\bar{\theta}=0.00$ & $\widehat{V}=0.13$ & $\widehat{V}=0.10$ & $\widehat{V}=0.002$ \\
\hline & & $\widehat{p}=0.79$ & $\widehat{p}=0.59$ & $\widehat{p}=0.40$ & $\widehat{p}=1.03$ & $\widehat{p}=0.98$ & $\widehat{p}=0.69$ \\
\hline & & & $\widehat{\beta}=2.03$ & - - & $\widehat{a}=1.15$ & - - & $\widehat{a}=0.28$ \\
\hline & & & $\widehat{d}=0.68$ & & & $\widehat{\beta}=0.87$ & $\widehat{\beta}=0.28$ \\
\hline & & & $T=1.05$ & & & & \\
\hline
\end{tabular}

\subsection{Borrowing library books}

The third example shows the number of books that were borrowed $k$ times $(k \geq 1)$ from the long loan collection at Sussex University over the period of a year (Burrell and Cane 1982). For fitting to this dataset, we consider the zero-truncation of each distribution. Table 3 shows the fitting results. From $\chi^{2}$ goodness of fit and AIC, the zero-truncation of the ECOMP distribution gives best fitting among fitted distributions. Studying the values of $\Delta_{i}$ suggest that the COM-NB distribution also has good support (evidence) while rest of the models have considerably less support for the data.. Here we interpret the size of queue in Section 2.6.1 as the popularity of books. Then, from the estimated parameters of the ECOMP distribution, we see that new interest is hard to increase but the popularity is hard to decrease for the book which is borrowed many times. This might be because, according as a book is borrowed more times, there are fewer opportunities to borrow the book. 
Table 3 The number of books that were borrowed $k$ times (Burrell and Cane 1982)

\begin{tabular}{|c|c|c|c|c|c|c|c|}
\hline Count & Observed & NB & Poi-GE & COMP & COMNB & GCOMP & ECOMP \\
\hline 1 & 9647 & 9522.59 & 9557.15 & 9364.75 & 9604.38 & 9576.21 & 9648.57 \\
\hline 2 & 4351 & 4595.67 & 4538.89 & 4706.62 & 4401.32 & 4458.54 & 4341.37 \\
\hline 3 & 2275 & 2296.39 & 2296.51 & 2365.50 & 2314.68 & 2311.93 & 2291.26 \\
\hline 4 & 1250 & 1167.09 & 1179.55 & 1188.87 & 1231.23 & 1213.94 & 1244.90 \\
\hline 5 & 663 & 599.12 & 608.51 & 597.51 & 643.23 & 630.96 & 663.27 \\
\hline 6 & 355 & 309.61 & 313.75 & 300.31 & 327.22 & 322.40 & 339.49 \\
\hline 7 & 154 & 160.75 & 161.73 & 150.93 & 161.73 & 161.61 & 165.46 \\
\hline 8 & 72 & 83.76 & 83.27 & 75.86 & 77.67 & 79.45 & 76.51 \\
\hline 9 & 37 & 43.76 & 42.82 & 38.12 & 36.28 & 38.33 & 33.54 \\
\hline 10 & 14 & 22.91 & 22.00 & 19.16 & 16.50 & 18.16 & 13.95 \\
\hline 11 & 6 & 12.02 & 11.28 & 9.63 & 7.32 & 8.46 & 5.51 \\
\hline 12 & 2 & 6.31 & 5.78 & 4.84 & 3.17 & 3.87 & 2.07 \\
\hline 13 & 0 & 3.32 & 2.96 & 2.43 & 1.34 & 1.75 & 0.74 \\
\hline 14 & 1 & 3.89 & 3.28 & 2.46 & 0.93 & 1.37 & 0.56 \\
\hline Total & 18,827 & 18827.00 & 18827.00 & 18827.00 & 18827.00 & 18827.00 & 18827.00 \\
\hline AlC & & 52461.94 & 52453.14 & 52472.22 & 52414.70 & 52422.75 & 52411.67 \\
\hline$x^{2}$ & & 51.96 & 38.33 & 66.83 & 7.20 & 14.75 & 2.37 \\
\hline$p$-value & & 0.00 & 0.00 & 0.00 & 0.51 & 0.06 & 0.94 \\
\hline \multirow[t]{5}{*}{ MLES } & & $\widehat{r}=0.81$ & $\widehat{v}=0.27$ & $\widehat{\theta}=0.00$ & $\widehat{v}=0.01$ & $\widehat{V}=0.01$ & $\widehat{v}=2.30$ \\
\hline & & $\widehat{p}=0.53$ & $\widehat{p}=0.38$ & $\widehat{p}=0.50$ & $\widehat{p}=1.18$ & $\widehat{p}=0.93$ & $\widehat{p}=6.40$ \\
\hline & & & $\widehat{\beta}=1.78$ & -- & $\widehat{a}=1.37$ & - - & $\widehat{a}=-2.98$ \\
\hline & & & $\widehat{d}=1.16$ & & & $\bar{\beta}=0.74$ & $\bar{\beta}=-3.95$ \\
\hline & & & $T=1.03$ & & & & \\
\hline
\end{tabular}

\section{Concluding remarks}

Extended Conway-Maxwell-Poisson distribution proposed here unifies the COM-NB and GCOMP which were recently introduced to add more flexibility to the COMPoisson distribution. The proposed distribution with additional parameter has more flexibility in terms of its tail behavior and dispersion level. Further it also arises from queuing theory set up and as exponential combination of negative binomial and COMPoisson distribution and has many interesting properties. It is therefore envisaged that ECOMP distribution has the potential in modeling varieties of count data.

\section{Appendix}

A. Approximations of the normalizing constant ${ }_{1} S_{a-1}^{\beta}(v ; 1 ; p)$ and the mean

\section{A.1 Asymptotic approximation of the normalizing constant ${ }_{1} S_{a-1}^{\beta}(v ; 1 ; p)$ using the} Laplace's method

Defining $i=\sqrt{-1}$, we have the identity for non-negative integers $n$ and $k$

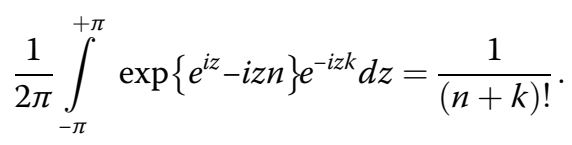

This leads to the identities 


$$
\begin{aligned}
& \frac{1}{2 \pi} \int_{-\pi}^{+\pi} \exp \left\{e^{i z}\right\}_{1} S_{a-1}^{\beta}\left(v ; 1 ; p e^{i z}\right) d z={ }_{1} S_{a}^{\beta}(v ; 1 ; p) \text { and } \\
& \frac{1}{2 \pi} \int_{-\pi}^{+\pi} \exp \left\{e^{i z}-i z(v-1)\right\}\{\Gamma(v)\}_{1}^{\beta} S_{a-1}^{\beta}\left(v ; 1 ; p e^{i z}\right) d z=\{\Gamma(v)\}^{\beta-1}{ }_{1} S_{a-1}^{\beta-1}(v ; 1 ; p)
\end{aligned}
$$

From these two identities, we get the formula for integer values $\alpha \geq 0$ and $\beta \leq 0$

$$
\begin{aligned}
{ }_{1} & S_{a-1}^{\beta}(v ; 1 ; p)\{\Gamma(v)\}^{\beta} \\
& =\frac{1}{(2 \pi)^{\alpha-\beta-1}} \int_{-\pi}^{+\pi} \cdots \int_{-\pi}^{+\pi} \exp \left\{\sum_{l=1}^{\alpha-\beta-1} e^{i z_{1}}+p e^{-\sum_{l=1}^{\alpha-\beta-1} i z_{l}}-(\nu-1) \sum_{l=1}^{\alpha-\beta-1} i z_{l}\right\} d z_{1} \cdots d z_{\alpha-\beta-1}
\end{aligned}
$$

Changing the variables $i z_{l}=i x_{l}+\log p /(\alpha-\beta)$ and then applying the Laplace's method for approximation of multiple integral, we obtain the formula (9).

The formula (9) has been derived for an integer values $\alpha \geq 1$ and $-\beta \geq 0$, but numerical studies suggest that it holds for $0<\alpha-\beta<1$ and $p>1$, where it is difficult to compute ${ }_{1} S_{a-1}^{\beta}(v, 1, p)$ by truncated approximation (8). The Table 4 gives the percentage errors $100\left\{\widetilde{S}_{1}^{\beta}{ }_{a-1}(v ; 1 ; p){ }_{-1} S_{a-1, m}^{\beta}(v ; 1 ; p)\right\} /{ }_{1} S_{a-1, m}^{\beta}(v ; 1 ; p)$, with $m=18000$ such that $R_{m}(v, p, \alpha, \beta)<10^{-28}$, where $\widetilde{S}_{a-1}^{\beta}(v ; 1 ; p)$ is the r.h.s. of the formula (9)

\section{A.2 Asymptotic approximation of the mean}

A numerical illustration of the performance of the asymptotic approximation formula of mean in equation (12) is provided in Table 5.

\section{B. Proof of the propositions}

\section{B.1 Proof of proposition 1}

Following Conway and Maxwell (1962), the system differential difference equations are given by

$$
P_{0}(t+\Delta)=\left(1-\lambda v^{\beta} \Delta\right) P_{0}(t)+\mu \Delta P_{1}(t)
$$

and

Table 4 The percentage errors for approximation (9) with $\beta=2.5$

\begin{tabular}{lllllllllll}
\hline \multicolumn{7}{c}{$v=0.5$} & \multicolumn{7}{c}{$v=1.5$} \\
\hline$p \backslash a$ & 2.6 & 2.7 & 2.8 & 2.9 & 3.0 & 2.6 & 2.7 & 2.8 & 2.9 & 3.0 \\
1.0 & 37 & 2 & -14 & -24 & -31 & -88 & -80 & -73 & -68 & -31 \\
1.2 & -57 & -44 & -42 & -43 & -44 & -55 & -61 & -60 & -58 & -44 \\
1.4 & -49 & -58 & -54 & -52 & -51 & -21 & -42 & -47 & -48 & -51 \\
1.6 & -8 & -55 & -57 & -55 & -54 & -7 & -28 & -37 & -40 & -54 \\
1.8 & -2 & -38 & -54 & -55 & -55 & -2 & -18 & -28 & -33 & -55 \\
2.0 & -1 & -18 & -46 & -52 & -54 & -1 & -11 & -22 & -28 & -54 \\
2.2 & 0 & -9 & -34 & -47 & -51 & 0 & -7 & -17 & -23 & -51 \\
2.4 & 0 & -5 & -23 & -40 & -47 & 0 & -5 & -13 & -20 & -47 \\
\hline
\end{tabular}


Table 5 The percentage errors for mean with $\beta=2.5$ using the approximation formula in equation (12)

\begin{tabular}{lllllllllll}
\hline & $v=0.5$ & & & \multicolumn{7}{c}{$v=1.5$} \\
\hline$p \backslash a$ & 2.6 & 2.7 & 2.8 & 2.9 & 3.0 & 2.6 & 2.7 & 2.8 & 2.9 & 3.0 \\
1.0 & -1055 & -731 & -575 & -475 & -403 & 107 & 78 & 64 & 55 & 48 \\
1.2 & -187 & -290 & -276 & -249 & -221 & 30 & 39 & 38 & 36 & 34 \\
1.4 & 46 & -40 & -93 & -107 & -106 & 5 & 18 & 23 & 24 & 24 \\
1.6 & 1 & 42 & 2 & -24 & -35 & 1 & 8 & 14 & 16 & 17 \\
1.8 & 0 & 28 & 36 & 19 & 6 & 0 & 3 & 8 & 11 & 13 \\
2.0 & 0 & 7 & 35 & 35 & 27 & 0 & 1 & 5 & 8 & 10 \\
2.2 & 0 & 1 & 22 & 35 & 34 & 0 & 1 & 3 & 6 & 7 \\
2.4 & 0 & 0 & 11 & 29 & 35 & 0 & -0 & 2 & 4 & 6 \\
\hline
\end{tabular}

$$
\begin{aligned}
P_{k}(t+\Delta)= & \left(1-\lambda(v+k)^{\beta} \Delta-\mu k^{\alpha} \Delta\right) P_{k}(t)+\lambda(v+k-1)^{\beta} \Delta P_{k-1}(t) \\
& \left.+\mu(k+1)^{\alpha} \Delta P_{k+1}(t)\right)
\end{aligned}
$$

Let $\lambda / \mu=p$. Then from (14) and (15) we get

$$
\begin{aligned}
\left\{P_{0}(t+\Delta)-P_{0}(t)\right\} / \Delta= & -\mu p v^{\beta} P_{0}(t)+\mu P_{1}(t) \text { and } \\
\left\{P_{k}(t+\Delta)-P_{k}(t)\right\} / \Delta= & \mu p(v+k-1)^{\beta} P_{k-1}(t)-\mu\left(p(v+k)+k^{\alpha}\right) P_{k}(t) \\
& \left.+\mu(k+1)^{\alpha} P_{k+1}(t)\right), k=1,2, \cdots
\end{aligned}
$$

Now as $\Delta \rightarrow 0$ we get

$$
\begin{aligned}
& \left.P_{0} /(t)\right\}=-\mu p v^{\beta} P_{0}(t)+\mu P_{1}(t) \text { and } \\
& \left.P_{k} /(t)=\mu p(v+k-1)^{\beta} P_{k-1}(t)-\mu\left(p(v+k)^{\beta}+k^{\alpha}\right) P_{k}(t)+\mu(k+1)^{\alpha} P_{k+1}(t)\right), \\
& \quad k=1,2, \cdots
\end{aligned}
$$

Assuming a steady state (i.e. $P_{k}^{\prime}(t)=0$ for all $k$ ) we get

$$
\begin{aligned}
& P_{1}(t)=p v^{\beta} P_{0}(t) \text { and } \\
& P_{k+1}(t)=\frac{k^{\alpha}}{(k+1)^{\alpha}} P_{k}(t)-\frac{(v+k)^{\beta}}{(k+1)^{\alpha}} p P_{k}(t)+\frac{(v+k-1)^{\beta}}{(k+1)^{\alpha}} p P_{k+1}(t), k=1,2, \cdots
\end{aligned}
$$

Putting $k=1$ we get

$$
\begin{aligned}
P_{2}(t) & =\frac{1}{2^{\alpha}} P_{1}(t)-\frac{(v+1)^{\beta}}{2^{\alpha}} p P_{1}(t)+\frac{v^{\beta} z}{2^{\alpha}} p P_{0}(t) \\
& =\frac{1}{2^{\alpha}} v^{\beta} p P_{0}(t)-\frac{(v+1)^{\beta}}{2^{\alpha}} v^{\beta} p P_{0}(t)+\frac{v^{\beta} z}{2^{\alpha}} p P_{0}(t) \\
& =\frac{\{v(v+1)\}^{\beta}}{(2 !)^{\alpha}} p^{2} P_{0}(t)=\frac{\left\{(v)_{2}\right\}^{\beta}}{(2 !)^{\alpha}} p^{2} P_{0}(t)
\end{aligned}
$$

Similarly, for $k=2$ we get 


$$
\begin{aligned}
P_{3}(t) & =\left(\frac{2^{\alpha}}{3^{\alpha}}-\frac{(v+2)^{\beta}}{3^{\alpha}} p\right) P_{2}(t)+\frac{(v+1)^{\beta} p}{3^{\alpha}} P_{1}(t) \\
& =\left(\frac{2^{\alpha}}{3^{\alpha}}-\frac{(v+2)^{\beta}}{3^{\alpha}} p\right) \frac{\{v(v+1)\}^{\beta}}{(2 !)^{\alpha}} p^{2} P_{0}(t)+\frac{(v+1)^{\beta} z}{3^{\alpha}} v p P_{0}(t) \\
& =\frac{\{v(v+1)(v+2)\}^{\beta}}{(3 !)^{\alpha}} p^{3} P_{0}(t)
\end{aligned}
$$

In general, $P_{k}(t)=\frac{\left\{(v)_{k}\right\}^{\beta}}{(k !)^{\alpha}} p^{k} P_{0}(t)$, where $P_{0}(t)=1 / \sum_{i=0}^{\infty}\left\{\frac{\left\{(v)_{i}\right\}^{\beta}}{(i !)^{\alpha}} p^{i}\right\}$.

Since we have assumed a steady state (i.e. $P_{k}^{/}(t)=0$ for all $k$ ) $P_{k}(t)$ can be replaced by $P_{k}$.

\section{B.2 Proof of proposition 2}

The probability function resulting from the exponential combination of NB $(v, \lambda)$ and COM-Poisson $(\mu, \theta)$ is given by

$$
\begin{aligned}
& \left\{\frac{(v)_{k}}{k !} \lambda^{k}\right\}^{\beta}\left\{\frac{\mu^{k}}{(k !)^{\theta}}\right\}^{1-\beta} / \sum_{i \geq 0}\left\{\frac{(v)_{i}}{i !} \lambda^{i}\right\}^{\beta}\left\{\frac{\mu^{i}}{(i !)^{\theta}}\right\}^{1-\beta} \\
& =\frac{\left\{(v)_{k}\right\}^{\beta}\left\{\lambda^{\beta} \mu^{1-\beta}\right\}^{k}}{(k !)^{\theta(1-\beta)+\beta}} / \sum_{i \geq 0} \frac{\left\{(v)_{i}\right\}^{\beta}\left\{\lambda^{\beta} \mu^{1-\beta}\right\}^{i}}{(i !)^{\theta(1-\beta)+\beta}}=\frac{\left\{(v)_{k}\right\}^{\beta} p^{k}}{(k !)^{\alpha}} / \sum_{i \geq 0} \frac{\left\{(v)_{i}\right\}^{\beta} p^{i}}{(i !)^{\alpha}}
\end{aligned}
$$

substituting $\lambda^{\beta} \mu^{1-\beta}=p$ and $\alpha=\theta(1-\beta)+\beta$

This is the pmf of ECOMP $(v, p, \alpha, \beta)$.

\section{B.3 Proof of proposition 3}

For a distribution to be log-concave we must have (see Gupta et al. 1997)

$$
\Delta \eta(t)=P(t+1) / P(t)-P(t+2) / P(t+1)>0 .
$$

For $\operatorname{ECOMP}(v, p, \alpha, \beta), \Delta \eta(t)=p \frac{(v+t)^{\beta}(t+2)^{\alpha}-(v+t+1)^{\beta}(t+1)^{\alpha}}{(t+1)^{\alpha}(t+2)^{\alpha}}$

$$
\begin{aligned}
& \text { Now } p \frac{(v+t)^{\beta}(t+2)^{\alpha}-(v+t+1)^{\beta}(t+1)^{\alpha}}{(t+1)^{\alpha}(t+2)^{\alpha}}>0 \\
& \Rightarrow(v+t)^{\beta}(t+2)^{\alpha}-(v+t+1)^{\beta}(t+1)^{\alpha}>0 \quad \text { since } \quad(t+1)^{\alpha}(t+2)^{\alpha}>0, p>0 \\
& \Rightarrow(v+t)^{\beta}(t+2)^{\alpha}-(v+t+1)^{\beta}(t+1)^{\alpha}>0 \\
& \text { But for } v>1, \quad(t+1) /(t+2)<(v+t) /(v+t+1) \\
& \Rightarrow\{(t+1) /(t+2)\}^{\alpha}<\{(v+t) /(v+t+1)\}^{\alpha} \leq\{(v+t) /(v+t+1)\}^{\beta} \quad \text { since } \alpha \geq \beta \\
& \Rightarrow\{(t+1) /(t+2)\}^{\alpha}\{(v+t+1) /(v+t)\}^{\beta}<1 \\
& \Rightarrow 1-\{(t+1) /(t+2)\}^{\alpha}\{(v+t+1) /(v+t)\}^{\beta}>0 \\
& \Rightarrow(v+t)^{\beta}(t+2)^{\alpha}-(v+t+1)^{\beta}(t+1)^{\alpha}>0
\end{aligned}
$$

\section{B.4 Proof of proposition 4}

ECOMP $(v, p, \alpha, \beta)$ has a log-convex probability mass function if $\Delta \eta(t) \leq 0$. That is 


$$
\begin{aligned}
& (v+t)^{\beta}(t+2)^{\alpha}<(v+t+1)^{\beta}(t+1)^{\alpha} \\
& \Rightarrow\{(t+2) /(t+1)\}^{\alpha}<\{(v+t+1) /(v+t)\}^{\beta} \\
& \Rightarrow\left\{(1+1 /(t+1)\}^{\alpha}<\{1+1 /(v+t)\}^{\beta}\right.
\end{aligned}
$$

Since $\alpha \geq \beta$ the inequality in (16) cannot hold for $v>1$.

Now for $0<v \leq 1$ the inequality in (16) implies

$$
\Rightarrow \alpha / \beta \leq \log (1+1 / t+v) / \log (1+1 / t+1) \geq 1
$$

$$
\Rightarrow \alpha / \beta \leq 1 \text {. Which implies } \alpha=\beta \text { since } \alpha \geq \beta \text {. }
$$

\section{Competing interests}

The authors declare that they have no competing interests.

\section{Authors' contributions}

SC conceptually developed the proposed distribution with related mathematical results of the paper and drafted the manuscript. TI developed the Sections 2.2, 2.8 and 4 of the manuscript. Both authors read and approved the final manuscript.

\section{Acknowledgments}

The corresponding author Prof. Subrata Chakraborty would like to thank the Editors -in-Chief Prof. Felix Famoye andProf. Carl Lee, for the invitation to write a paper for this esteemed Journal. Both the authors acknowledge the comments and suggestions of the editor and both the reviewers which lead to substantial improvement in the presentation of the work.

\section{Author details \\ ${ }^{1}$ Department of Statistics, Dibrugarh University, Dibrugarh 786004, Assam, India. ${ }^{2}$ The Institute of Statistical Mathematics, 10-3 Midori-cho, Tachikawa, Tokyo 190-8562, Japan.}

Received: 10 October 2015 Accepted: 9 February 2016

Published online: 25 February 2016

\section{References}

Abramowitz, M., Stegun, I.A.: Handbook of Mathematical Functions. $9^{\text {th }}$ Print. Dover, New York (1970)

Agarwal, S.K., Kalla, S.L.: A generalized gamma distribution and its application in reliability. Commun. Stat. Theory Methods $25,1,201-210(1996)$

Atkinson, A.C.: A method for discriminating between models. J. R. Stat. Soc. Series B (Methodological) 32, 3, 323-353 (1970) Bleistein, N., Handelsman, R.A.: Asymptotic expansions of integrals. Dover, New York (1986)

Burnham, K.P., Anderson, D.R.: Multimodel Inference-Understanding AIC and BIC in Model Selection. Sociol. Methods Res. 33, 2, 261-304 (2004)

Burrell, Q.L., Cane, V.R.: The analysis of library data. J. R. Stat. Soc., Series A 145, 439-471 (1982)

Chakraborty, S., Ong, S.H. A COM-type generalization of the negative binomial distribution, Accepted in April 2014, (available on line since 07 November 2015) to appear in Communications in Statistics-Theory and Methods Conway, R.W., Maxwell, W.L.: A queueing model with state dependent service rates. J Industrl Engng 12, 132-136 (1962)

Corbet, A.S.: The distribution of butteries in the Malay peninsula. Proc. R. Entomol. Soc. London, Series A, General Entomology 16, 101-116 (1942)

Cox, D.R.: Tests of separate families of hypotheses. Proc. 4th Berkeley Symp. 1, 105-123 (1961)

Cox, D.R.: Further results on tests of separate families of hypotheses. J. R. Statist. Soc. B 24, 406-424 (1962)

Gómez-Déniz, E., María Sarabia, J., Calderín-Ojeda, E.: A new discrete distribution with actuarial applications. Insur. Math. Econ. 48, 406-412 (2011)

Gupta, R.C., Ong, S.H.: Analysis of long-tailed count data by Poisson mixtures. Commun. Stat. Theory Methods 34, 557-574 (2005)

Gupta, P.L., Gupta, R.C., Tripathi, R.C.: On the monotonic properties of discrete failure rates. J. Stat. Plan. Inference 65, 255-268 (1997)

Gupta, R.C., Sim, S.Z., Ong, S.H.: Analysis of discrete data by Conway-Maxwell Poisson distribution. AStA Adv. Stat. Anal. 98, 327-343 (2014)

Imoto, T:: A generalized Conway-Maxwell-Poisson distribution which includes the negative binomial distribution. Appl. Math. Comput. 247, 824-834 (2014)

Johnson, N.L., Kemp, A.W., Kotz, S.: Univariate discrete distributions. Wiley, New York (2005)

Jorgensen, B.: Statistical properties of the generalized inverse Gaussian distribution. Lecture Notes in Statistics, SpringerVerlag, New York (1982)

Kokonendji, C.C., Mizère, D., Balakrishnan, N.: Connections of the Poisson weight function to over dispersion and unde rdispersion. J. Stat. Plan. Inference 138, 1287-1296 (2008)

Lin, S-K.: Characterization of lightning as a disturbance to the forest ecosystem in East Texas. M.Sc. thesis. Texas A \& M University, College Station (1985) 
Minka, T.P., Shmueli, G., Kadane, J.B., Borle S., and Boatwright, P.: Computing with the COM-Poisson distribution. Technical Report: 776, Department of Statistics, Carnegie Mellon University, http://repository.cmu.edu/cgi/viewcontent. cgi?article $=1174 \&$ context=statistics . (2003)

Ong, S.H., Lee, P.A.: On a generalized non-central negative binomial distribution. Commun. Stat. Theory Methods 15, 1065-1079 (1986)

Shaked, M., Shanthikumar, J.G.: Stochastic orders. Springer Verlag, New York (2007)

Warde, W.D., Katti, S.K.: Infinite divisibility of discrete distributions II. Ann. Math. Stat. 42, 3, 1088-1090 (1971)

Submit your manuscript to a SpringerOpen ${ }^{\odot}$ journal and benefit from:

- Convenient online submission

- Rigorous peer review

- Immediate publication on acceptance

- Open access: articles freely available online

- High visibility within the field

- Retaining the copyright to your article

Submit your next manuscript at $\boldsymbol{\wedge}$ springeropen.com 